\title{
EMPIRICAL CADESTUDY ON APPROACHES TO SAMPLED CONTROL
}

\author{
Alexandra Burger and Jens Wellhausen \\ Jade University of Applied Sciences \\ Department of Engineering \\ Friedrich Paffrath Str 101, 26389 Wilhelmshaven \\ Germany \\ \{alexandra.burger, jens.wellhausen\}@jade-hs.de
}

\begin{abstract}
Two main approaches on sampled control are compared, the descrete equivalent design and the direct discrete design. Both design methods are used to implement a control system for a real plant, DC-motor. It is shown that discrete equivalent design works well with sufficient high sampling rates and that direct discrete design works nearly independent of sampling time.
\end{abstract}

Keywords: discrete control, PI-controller, state space control, observer, feedforward control, structure with two degrees of freedom

\section{Introduction}

There are mainly two approaches to sampled control [1]:

- discrete equivalent design (DED)

- direct discrete design (DDD)

In DED a continous time controller is calculated and afterwards approximated by an algorithm and sample and hold blocks. In DDD a matching time discrete description of the plant is calculated. For this difference equation a discete control algorithm is derived. Since in principle the DDD-method doesn't work with approximations, it is commonly known that this method works fine even for lower sampling rates, whereas the DED-method is restricted to sampling rates about 10 times higher than the model dynamics. The DED-method works well and is frequently used in industrial applications. The DDD-method is a more mathematical approach. It depends highly on the qualitiy of the mathematical model of the plant and its time discrete representation. For further information about the above described methods see [1]. The aim of this empirical study (setup see figure 1) is to determine how these two methods work with a real plant, a DC-motor of low power, which is represented by its linear approximation. In DED a PI-controller is used, whereas in DDD a state space controller in a structure with two degrees of freedom and feed forward is applied.

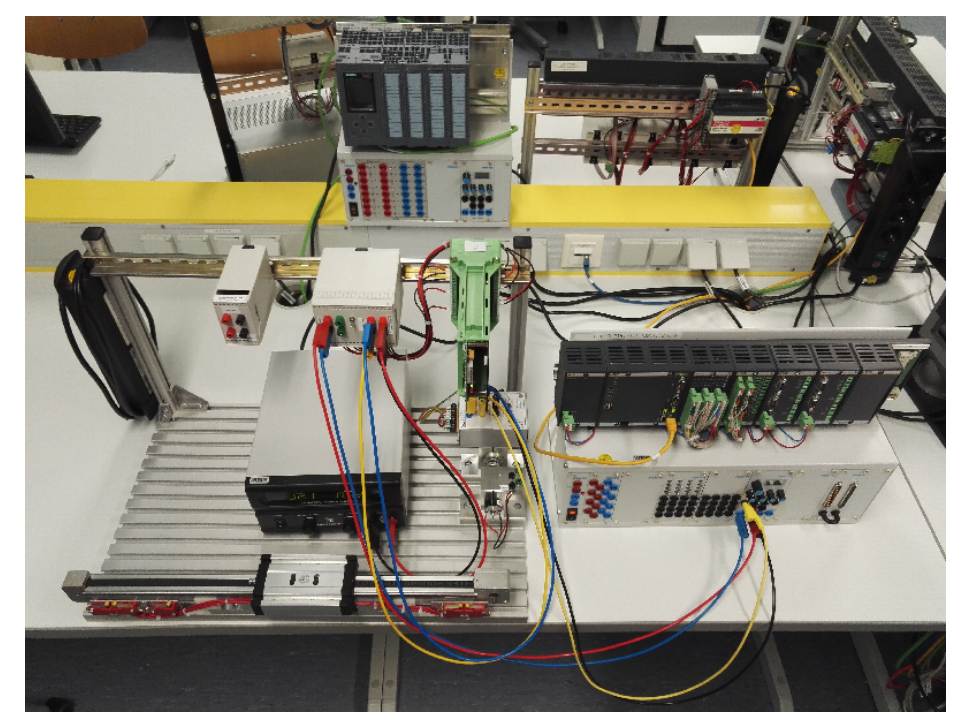

Figure 1: DC-Motor controlled by PLC 


\section{Experimental setup}

Figure 2 shows the control loop, consisting of plant (DC-motor), actuator (power electronics), sensor (tachogenerator) and the controller, run on a PLC. The controlled variable is angular speed. For the plant a linear second order model is used. The dynamics of the actuator and the sensor are omitted. Equations (1) and (2) represent the plant combined with actuator and sensor. For further information in modelling a DC-Motor see $[2],[3],[4]$.

$$
\begin{aligned}
\dot{x} & =A x+b u_{S T}+b_{1} M_{L} \\
\omega & =c x
\end{aligned}
$$

with $x:=\left[\begin{array}{c}i_{A} \\ \omega\end{array}\right], A:=\left[\begin{array}{cc}-\frac{R_{A}}{L_{A}} & -\frac{K_{\Psi}}{L_{A}} \\ \frac{K_{\Psi}}{J} & 0\end{array}\right], b:=\left[\begin{array}{c}\frac{K_{P S T}}{L_{A}} \\ 0\end{array}\right], b_{1}:=\left[\begin{array}{c}0 \\ \frac{1}{J}\end{array}\right], c:=\left[\begin{array}{ll}0 & 1\end{array}\right]$

Values for armature resistance $R_{A}$, armature inductivity $L_{A}$, mass moment of inertia $J$ and $K_{\Psi}$ can be obtained from the datasheet. The motor is described by the two state variables angular speed $\omega$ and armature current $i_{A}$. The angular speed is to be controlled by armature voltage $u_{S T}$, therefore it is an input to the state space differential equation. The second input is the momentum of load $M_{L}$ representing a disturbance. Friction is not considered in this setup.

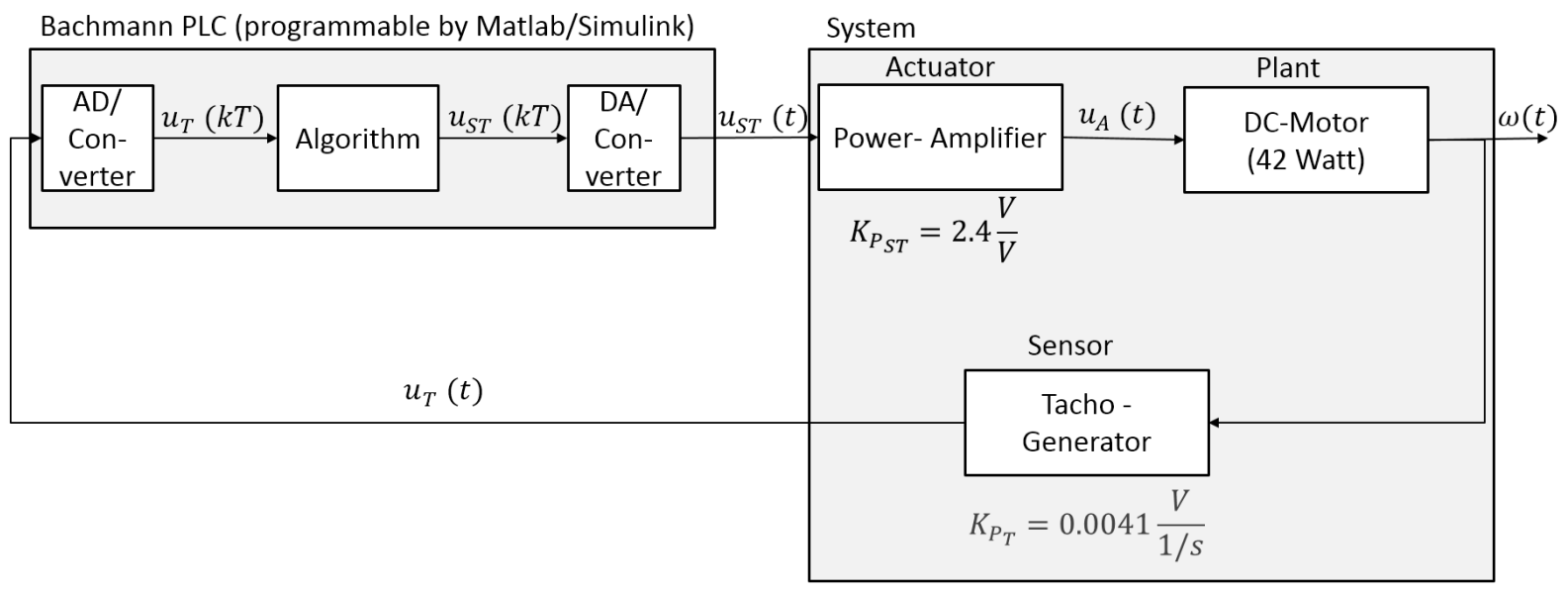

Figure 2: Setup of experiment

\section{Discrete equivalent design}

\subsection{Overview}

In discrete equivalent design the following steps must be performed [1]:

- a continuous time controller using time continous design techniques is derived

- a sampling rate lower then the dominant time constant of the continuous time closed loop system is choosen

- the controller $R(s)$ is discretized using an approximation of $z=e^{s T}$, wher $T$ is sampling time

- the algorithm is implemented on a platform

Advantage of this technique is, that all empirical continous time design methods can be used, e.g. for developing a PI-controller. Also, many PLCs come along with discrete PID algorithms, so the only task is to apply the continuous time parameters of the controller. Disadvantage is that the controller only holds for sufficient small sampling times. 


\subsection{Controller design}

For continuous time controller design, plant dynamics are approximated by a $P T_{1}$-transfer function (see [2], [4]), including proportionalities for actuator and sensor. A PI-controller was calculated for nearly overdamped behaviour. This leads to a controller transferfunction of

$$
R(s)=0.33 \frac{0.01 s+1}{0.01 s}
$$

and the closed loop transfer function

$$
R(s)=\frac{1}{K_{P T}} \frac{0.01 s+1}{\frac{1}{55^{2}} s^{2}+\frac{1.8}{55} s+1}
$$

The controller was discretized using Tustin's approximation with the sample time T

$$
s=\frac{2}{T} \frac{z-1}{z+1}
$$

and is described by the discrete transfer function

$$
R(z)=0.33\left(1+\frac{T}{0.02} \frac{z+1}{z-1}\right)
$$

\section{Direct discrete design}

\subsection{Overview}

For the DDD-method the following steps were performed [1]:

- describe the system by a sufficiently matching linear state space model

- calculate the solution of the continuous state space model for constant inputs (if zero order holds are used)

- take the solution at sample times and get the time discrete state space model

- use the discrete state space model to derive a discrete state space controller

If both, the continous time model and the solution of the differential equation are exact, the discrete time model is also exact. Therefore there is no limitation on the sample time.

\subsection{Controller design}

For the experiment, the linear state space repesentation of the plant (see equation (1)) is used and friction is omitted. It's time discrete representation is obtained by solving the continous time equation for constant inputs and taking the soloution at multiples of sampling times. This leads to a discrete time state space model

$$
\begin{aligned}
& x_{k+1}=F x_{k}+g u_{S T, k}+g_{1} M_{L, k} \\
& u_{T, k}=c x_{k}
\end{aligned}
$$

where

$$
\begin{gathered}
F=e^{A T} \\
g=\int_{0}^{T} e^{A(T-\tau)} b d \tau \\
g_{1}=\int_{0}^{T} e^{A(T-\tau)} b_{1} d \tau
\end{gathered}
$$

In DDD a state space controller approach was used. The structure of the control system is two degrees of freedom [5] and consists of an observer [6] feedback and feed forward parts for disturbance and referece input. Figure 3 shows the resulting control structure. The control law consists of the following parts: State feedback $r$ calculated by pole-placement of $(F+g r)$, feed forward $S_{u}$ and decoupling $S_{x}$

$$
\begin{aligned}
& S_{u}=\left[\left[\begin{array}{cc}
0 & 1
\end{array}\right](I-F)^{-1} g\right]^{-1} \\
& S_{x}=(I-F)^{-1} S_{u}
\end{aligned}
$$


To deal with disturbances, feed forward control for constant disturbances $L$ and decoupling $M$ is used.

$$
\begin{aligned}
L & =-\left[\left[\begin{array}{cc}
0 & 1
\end{array}\right](I-F)^{-1} g\right]^{-1}\left[\begin{array}{ll}
0 & 1
\end{array}\right](I-F)^{-1} g_{1} \\
M & =(I-F)^{-1}\left(g L+g_{1}\right)
\end{aligned}
$$

Since neither armature current for state feedback nor momentum of load for disturbance feed forward are mesured, an observer is implemented. The observer uses an extended model given by equation (13), for more information on disturbances on DC-motors see [8]. The extended model is observable, since the reduced model is observable and the disturbance model's eigenvalue differs from the eigenvalues of the DC-motor model. Since a linear modor dynamic was assumed, a linear observer is sufficient. For further information on local observability see $[7]$.

$$
\left[\begin{array}{c}
\hat{x}_{k+1} \\
\hat{M}_{L, k+1}
\end{array}\right]=\left[\begin{array}{cc}
F & g_{1} \\
0 & 1
\end{array}\right]\left[\begin{array}{c}
\hat{x}_{k} \\
\hat{M}_{L, k}
\end{array}\right]+\left[\begin{array}{c}
g_{1} \\
0
\end{array}\right] u_{S T, k}
$$

This model will only work for constant disturbances, which were assumed. Observer and feedback gain are choosen not to move the eigenvalues too far, because the control voltage is limited.

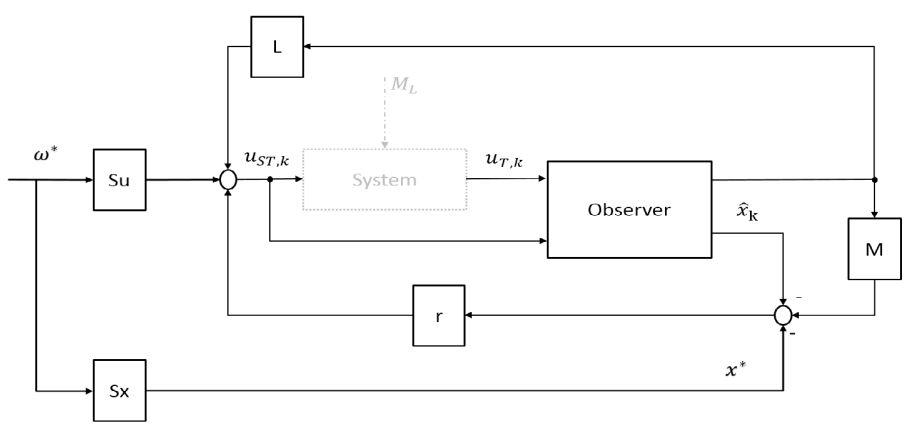

Figure 3: Control law DDD-method

\section{Results}

\subsection{PI controller, DED}

In an experimental setup the derived controller was implemented and tested with the real plant. Figure 4 shows the step responses of the system controlled with the PI-controller.

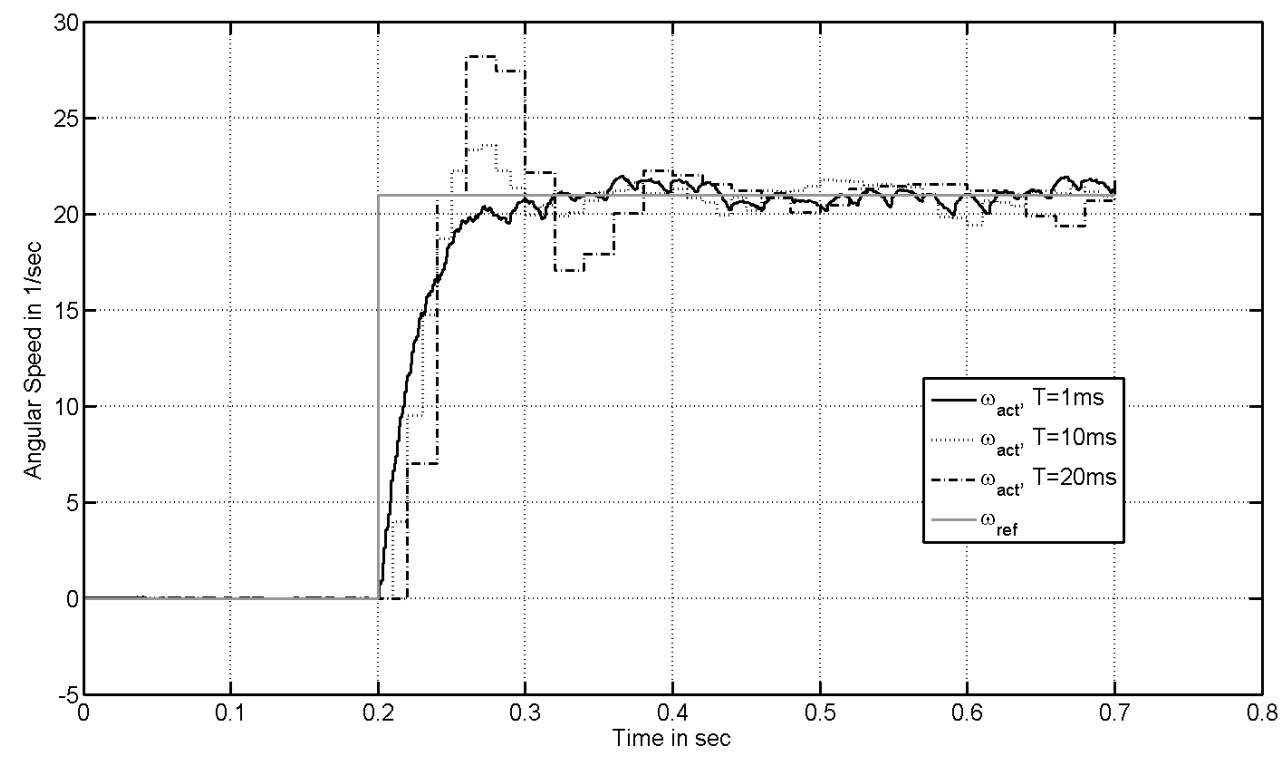

Figure 4: Step response, PI-controller, DED 
As expected it works fine for sufficient high sampling rates, as sampletime grows the controlled system tends to undemped behaviour. In this setup, a sample time greater than $40 \mathrm{msec}$ leads to instable behaviour, figure 5 .

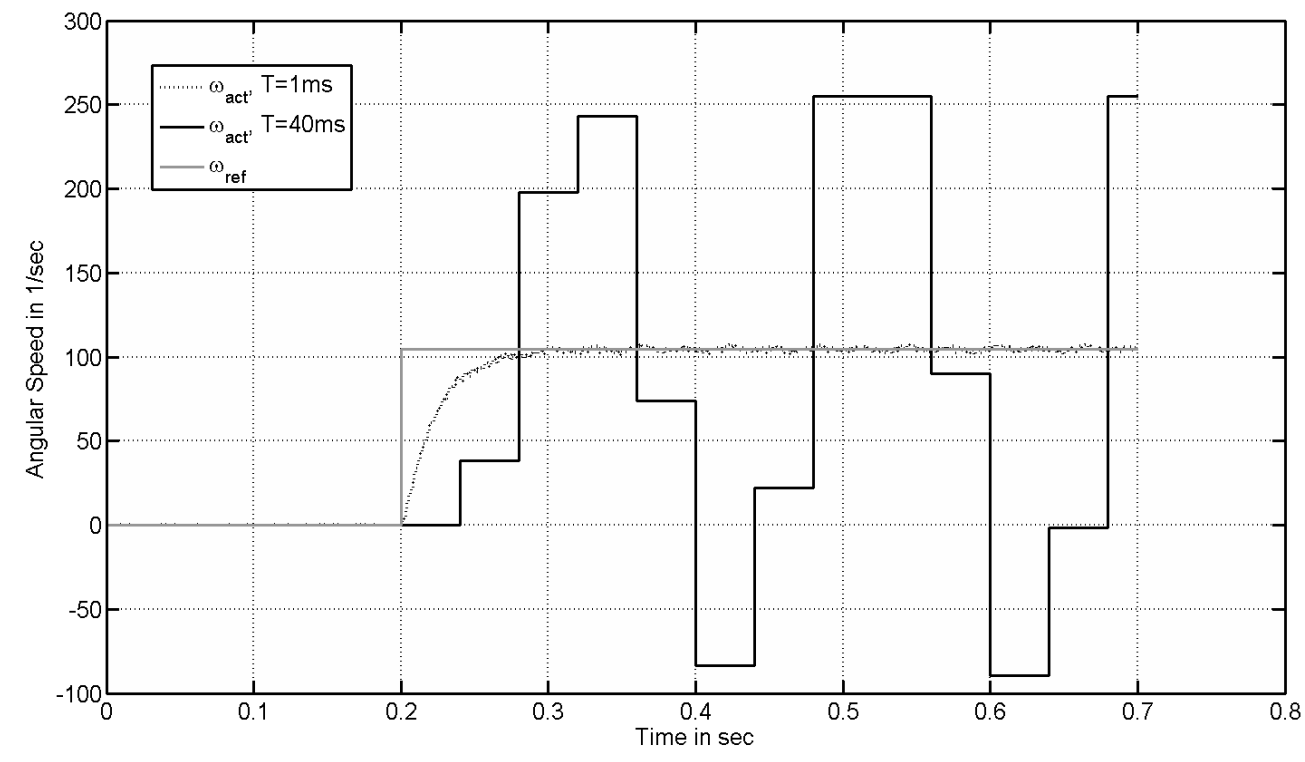

Figure 5: DED, instability due to sample time

\subsection{State space controller, DDD}

The control algorithm was implented on a PLC and run with the real plant. As Figure 6 shows, the step response converges to its setpoint even for higer sampling rates. So, for the DC-motor, the error caused by omitting nonlinearities and friction proves to be sufficient small. Figure 7 shows the actuation variable (voltage of the poweramplifier). For very small samplingtimes, a distinct overshoot can be seen. This overshoot diminishes, when samplingtime increases.

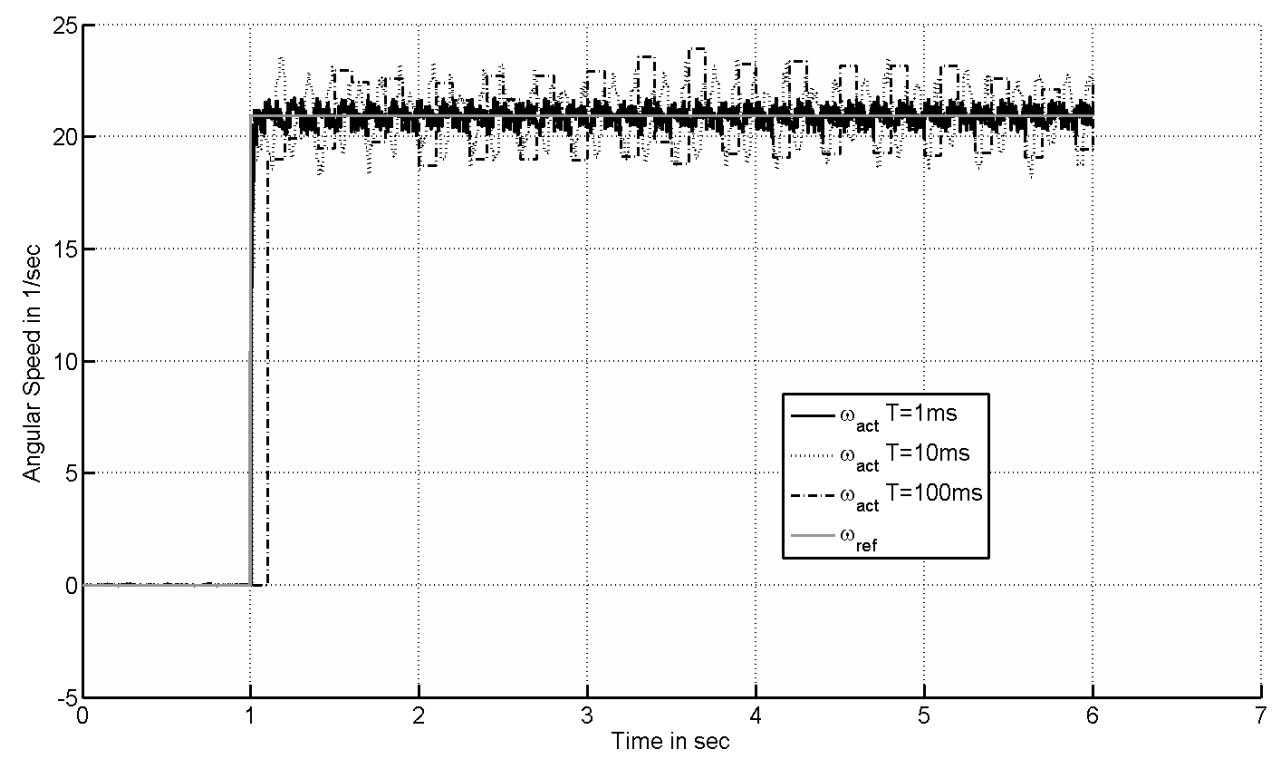

Figure 6: Step response state space controller, DDD 


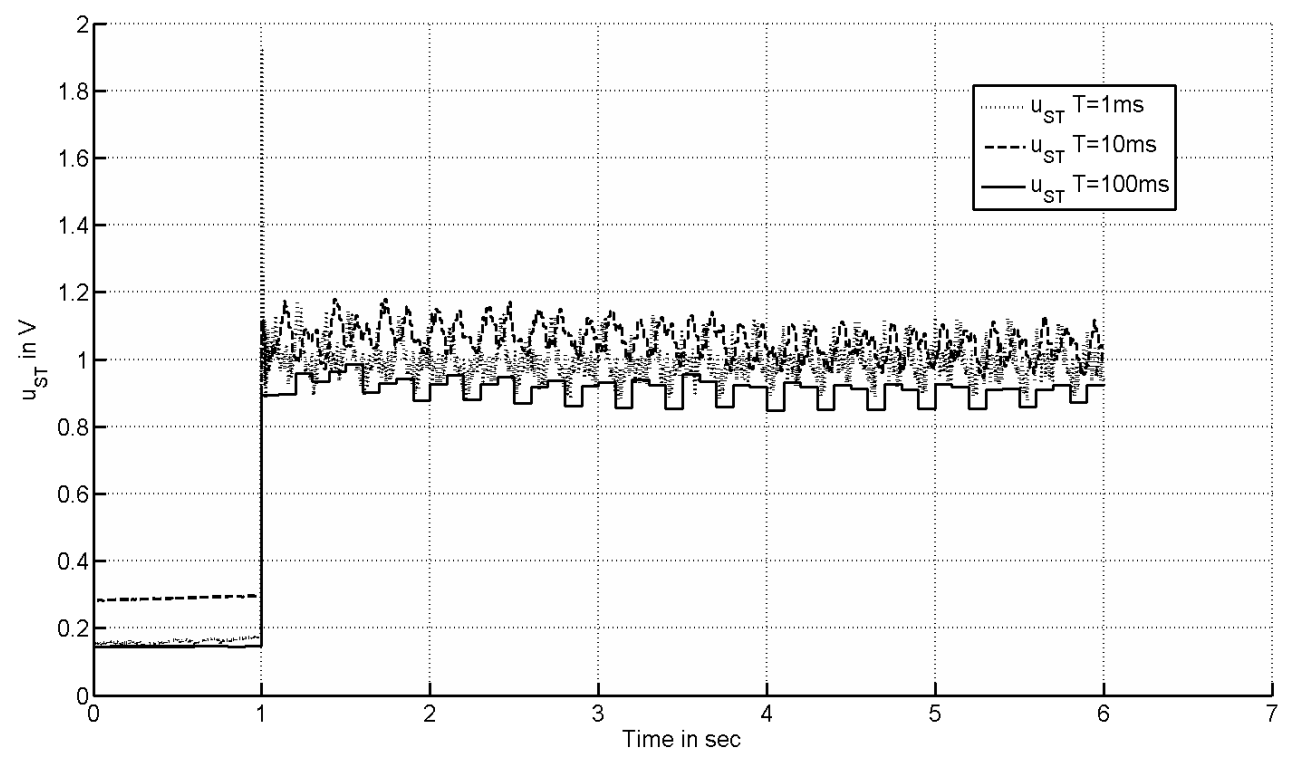

Figure 7: Control voltage generated by DDD controller

Figure 8 shows the difference between an observer and the extended observer. Since friction was omitted in the model, a basic observer calculates the angular speed as too high. An extended observer incorporating a disturbance model on the other hand is capable of compensating the model error caused by friction.

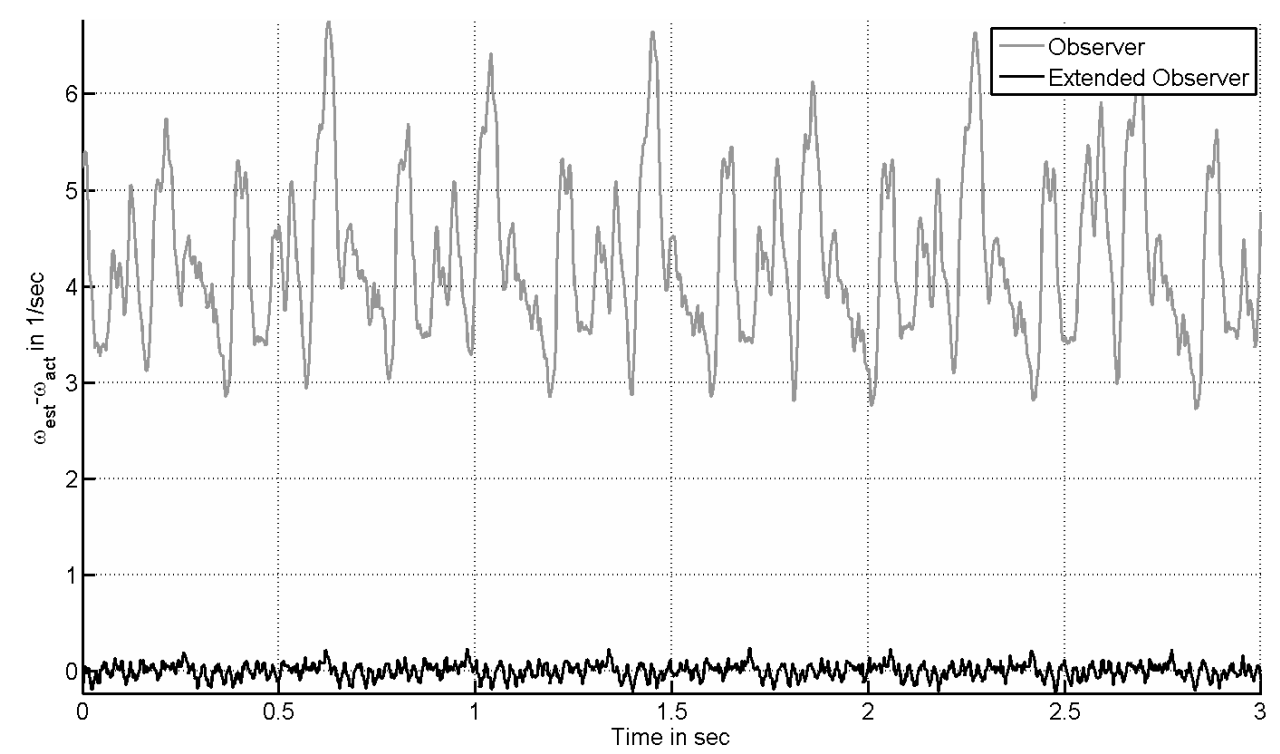

Figure 8: Observing error at angular speed of 20.9 1/sec, DDD 


\subsection{Comparision of DED and DDD-method}

As seen in figure 9, the DDD controller leads the controlled variable faster to its setpoint than the DED controller. This difference in settime is due to differences in polplacement. Figure 10 shows that the state space controller needs more armature voltage. This effect is lessend, when one uses lower sampling rates, as illustrated in figure 7 .

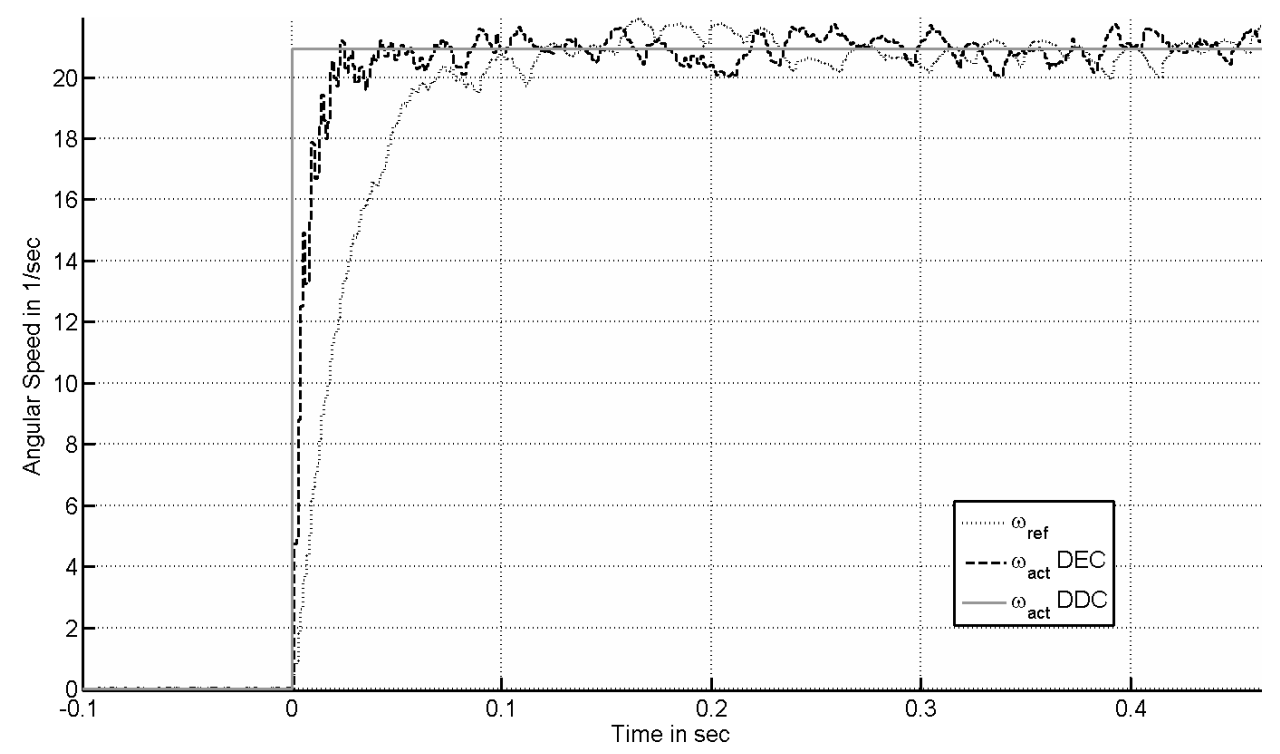

Figure 9: Step response DED controller and DDD controller at a sample time of $1 \mathrm{msec}$

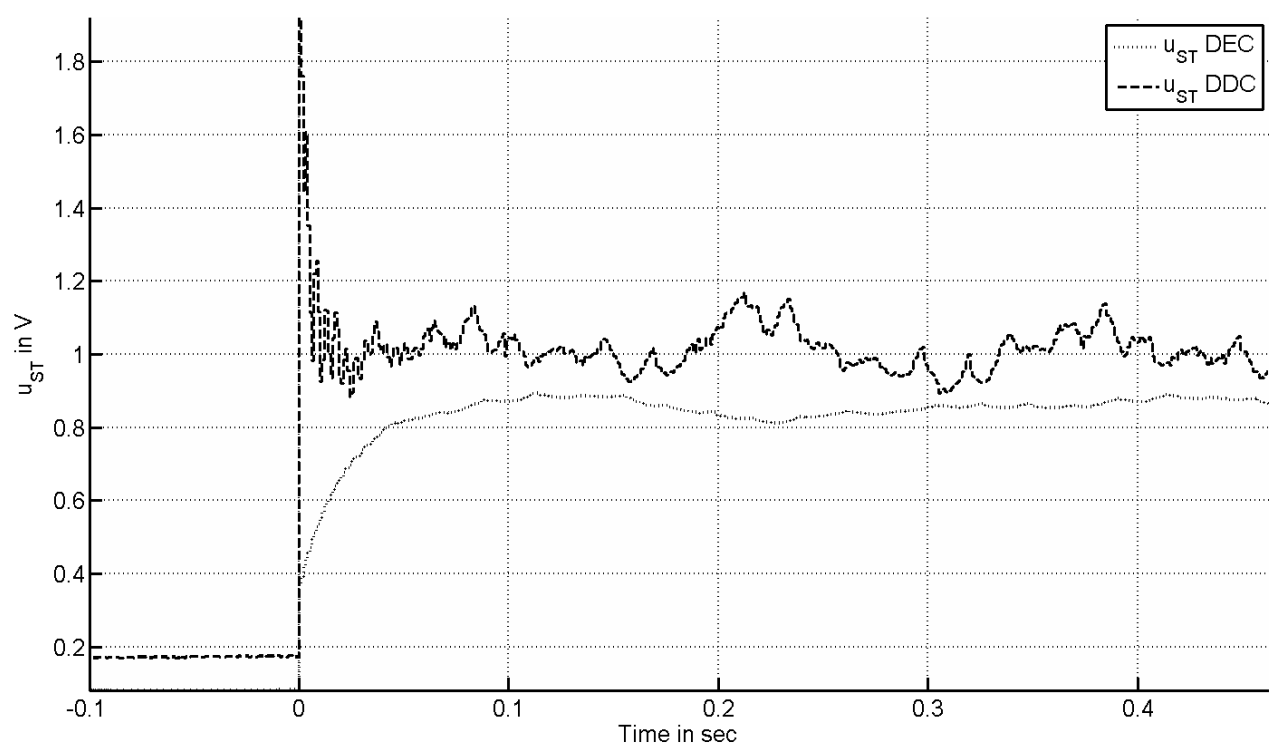

Figure 10: control voltage generated by DED controller and DDD controller at a sample time of $1 \mathrm{msec}$ 


\section{Conclusion}

In an experimental setup, two different techniques of discrete controller design were tested and compared. The discrete equivalent design technique, well known in various industrial applications, works sufficient for high enough sampling rates. Tending towards lower sampling rates the control loop shows increasing undempt behaviour up to the point of instability. For direct discrete controller design, the engineering costs are higher. A sufficiently matching discrete state space model must be calculated, which is difficult for many real world applications. Afterwards, when using a state space controller, an extended observer and disturbance feed forward is needed. The experiments showed, that the usual linear approximation of the DC-motor without friction is a sufficiently exact model. Although the DDD controller requires substantial additional effort in the design phase, it allows good controller behaviour at reduced sampling frequencies compared to the DED controller.

\section{References}

[1] Gene F. Franklin, J. David Powell, Abbas Emami-Naeini : Feedback Control of Dynamic Systems, sixth edn. Prentice-Hall, 2010, ISBN-10: 0136019692, ISBN-13: 9780136019695.

[2] D. Schroeder : Elektrische Antriebe - Grundlagen, forth edn. Springer, 2009, ISBN-10: 0937-7433, ISBN-13: 9783642029899.

[3] D. Schroeder : Elektrische Antriebe - Regelung von Antriebssystemen, forth edn. Springer, 2015, ISBN: 978364230095 .

[4] W. Leonhard Regelung elektrischer Antriebe, second edn. Springer, 2000, ISBN:356067179.

[5] Kreisselmeier, G., Struktur mit Zwei Freiheitsgraden, at - Automatisierungstechnik, ISSN (Online) 2196677X, 1.6.1999, DOI 10.1524/auto.1999.47.6.266

[6] Kreisselmeier, G., On sampling without loss of observability/controllability, IEEE Trans. Automat. Contr. 44(5): 1021-1025 (1999)

[7] Navrat, A., Matousek, R.: Trident snake control based on conformal geometric algebra. In Advances in Intelligent Systems and Computing, volume 378. Brno, Springer-Verlag, pp 375385. (2015). DOI: 10.1007/9783-319-19824-831

[8] Matousek, R.; Nemec, Z., Marada, T.: Weighting of goods by means of DC motor. In Advanced Material Research, volume 463-464. In 2nd International Conference on Advanced Material Research, ICAMR 2012, Chengdu, China, pp 14271431, (2012). DOI: 10.4028/www.scientific.net/AMR.463-464.142 